\title{
Anticoagulante circulante y lesiones focales hepáticas asociados a marcada semiología sistémica en la lúes secundaria
}

\author{
S. SUÁREZ ORTEGA, J. LÓPEZ BRITO ${ }^{1}$, H. MENDOZA LÉMES, C. R. HERNÁNDEZ \\ SOCORRO $^{2}$, E. MELADO SÁNCHEZ, J. DELGADO MARTÍNEZ
}

Servicios de Medicina Interna, ${ }^{1}$ Hematología y ${ }^{2}$ Radiología. Hospital General de Gran

Canaria Dr. Negrín. Las Palmas de Gran Canaria

CIRCULATING ANTICOAGULANT AND FOCAL LIVER LESIONS ASSOCIATED TO RICH CLINICAL EXPRESSION IN THE SECONDARY SYPHILLIS

\section{RESUMEN}

Un varón de 37 años ingresó en nuestro hospital por un cuadro de 8 semanas de evolución de malestar general, fiebre, artralgias, molestias faríngeas y nasales, cefalalgia holocraneal, fotofobia y náuseas. Con la ducha notó lesiones maculares en palmas de manos y plantas de pies. Un año antes había notado erosiones indoloras en el prepucio. Tenía factores de riesgo para enfermedades de transmisión sexual. La analítica mostró criterios de colestasis disociada, síndrome nefrótico, presencia de anticoagulante circulante, y positividad para la serología reagínica y específica de lúes. En una ecografía abdominal se objetivaron múltiples lesiones pequeñas, centimétricas, ocupantes de espacio. Con 2 semanas de tratamiento con penicilina revirtieron todas las manifestaciones clínicas, analíticas y de imagen. Se comenta la rica expresión semiológica y las peculiaridades de presentar lesiones ocupantes de espacio hepáticas y anticoagulante circulante en un caso de sífilis secundaria.

PALABRAS CLAVE: Sífilis secundaria. Hepatitis. Síndrome nefrótico. Anticoagulante circulante. Lesiones focales hepáticas.

\begin{abstract}
A 37-year-old male was admitted at our hospital for evaluation of clinical presentation of 8 weeks evolution of malaise, fever, sore throat and nose, arthralgias, holocraneal headache, photophobia and nausea. With the shower he noticed spots in palms of hands and plants of feet. A year before had noticed painless erosions in foreskin. He had risk factors for sexual transmission diseases. The analytical showed criteria of dissociated colestasis, nephrotic syndrome, presence of circulating anticoagulant, and positivity for the reaginic and specific serological syphilis. In an abdominal ultrasonic multiple, focal and small liver lesions were watched. With two weeks of treatment with penicillin the clinical manifestations reverted, and the analytical and of image was watched bettering, which dissapeared at the three montnths of treatment. We comment the rich clinical expression and the peculiarities of presenting focal liver lesions and circulating anticoagulant, in a case of secondary syphilis.
\end{abstract}

KEY WORDS: Secondary syphilis. Hepatitis. Nephrotic syndrome. Circulating anticoagulant. Focal liver diseases.

Suárez Ortega S, López Brito J, Mendoza Lémes, Hernández Socorro CR, Melado Sánchez E, Delgado Martínez J. Anticoagulante circulante y lesiones focales hepáticas asociados a marcada semiología sistémica en la lúes secundaria. An Med Interna (Madrid) 2007; 24: 331-334.

\section{INTRODUCCIÓN}

Las manifestaciones cutáneo-mucosas de la lúes secundaria suelen ocultar la expresión sistémica de dicha enfermedad, aunque pueden estar ausentes hasta en el $25 \%$ de los casos. Las manifestaciones sistémicas como fiebre, artralgias, meningitis, hepatitis, osteítis y síndrome nefrótico son menos frecuentes $(1,2)$, si bien existe un número considerable de casos publicados con síndromes aislados de esta semiología sistémica, que determina aproximadamente unas 80 citas bibliográficas en una búsqueda Medline (1960-2006). La semiología sistémica florida asociada a anticoagulante circulante es rara, como lo muestra una búsqueda en Medline, que representa sólo 3 casos, y un cuarto coexistiendo con fiebre Q (3-6). Coexistiendo con lesiones focales hepáticas (LOES) solamente existen 2 citas bibliográficas $(7,8)$. Recientemente hemos estudiado un paciente con clínica sistémica, consisten- te en fiebre, diátesis hemorrágica, artralgias, reacción meníngea, síndrome nefrótico y hepatitis colestática, en la que destaca junto a esa marcada semiología la presencia de LOES hepáticas y anticoagulante circulante, con rápida desaparición de todo el cuadro clínico tras tratamiento con penicilina, lo que es el motivo de esta presentación. Estas manifestaciones asociadas son descritas en la literatura de modo aislado (3-14), no existiendo hasta el momento actual en Medline ningún caso con toda la semiología comentada.

\section{CASO APORTADO}

Varón de 37 años, que encontrándose relativamente estacionario hacia finales de marzo del 2006 comenzó a notar náuseas, vómitos y dificultad para tragar de comienzo brusco, y una semana después quebrantamiento del estado general, mialgias en cintura escapular y 
artralgias en rodillas. Posteriormente aparece cefalea holocraneal esporádica, fotofobia, fiebre de hasta $38,5^{\circ} \mathrm{C}$ de predominio vespertino, congestión nasal y sensación de taponamiento ótico. Un mes antes del ingreso, en relación con una limpieza dental, presentó importante sangrado. En Mayo de 2006 se decidió ingreso hospitalario ante el cuadro clínico general junto a la presencia de colestasis, nódulos hepáticos, proteinuria en rango nefrótico y perfil clínico de reacción meníngea. Antecedentes personales de litiasis urinaria, habiéndose practicado litotripsia un año antes. Cuadro cutáneo descamativo de larga evolución que ha sido filiado como psoriasis. En los meses previos había notado máculas rojizas, evanescentes tras la ducha en palmas y plantas. Tabaquismo moderado. Factores de riesgo de enfermedades de transmisión sexual. A la exploración clínica: Impresiona de enfermedad aguda; febricular. No rigidez de cuello. Microadenopatías en cuello. Faringe enrojecida. Lesiones descamativas a nivel de codos y rodillas. Marcado hirsutismo. Auscultación cardio-pulmonar normal. TA 90/60 mm de $\mathrm{Hg}$. Hepatomegalia de 2 $\mathrm{cm}$, con dolorimiento a la presión. Resto sin hallazgos. De la analítica destaca: moderada leucocitocis $(15.200 \mathrm{l} / \mathrm{mcl})$ con linfocitosis (6.370 linfocitos (mcl). Plaquetas 480.000. Creatinina $1,31 \mathrm{mg} / \mathrm{dl}$; Colesterol total $377 \mathrm{mg} / \mathrm{dl}$; colesterol no-HDL $324 \mathrm{mg} / \mathrm{dl}$; TGO 116 (normal de 5-38 U/1), TGP 208 normal de 5-41 U/1), GGT 1.177 (normal de 7-50 U/l) y fosfatasas alcalinas $1.344 \mathrm{U} / \mathrm{l}$ (normal de 40$129 \mathrm{U} / \mathrm{l})$, bilirrubina total de $1,65 \mathrm{mg} / \mathrm{dl}$, con fracción directa de 0,95 $\mathrm{mg} / \mathrm{dl}$, con marcada mejoría a los diez días de tratamiento y normalización 3 meses después (Tabla I). Hormonas tiroideas, crioglobulinas, ceruloplasmina, alfa 1-antitripsina, cupremia, y resto de un protocolo con 22 determinaciones, virus B y virus $\mathrm{C}$ de la hepatitis, VIH 1 y 2 y serología de fiebre de origen desconocido: normal o negativo. TPHA positivo +++, con RPR 1/32. Hemocultivos negativos. Estudio de coagulación: tiempo parcial de tromboplastina activado (APTT) de 64 segundos, correspondiendo a un ratio de 1,81; tiempo de trombina (TT) de 24 segundos (VN: 13-22) y un tempo de reptilase (TR) dentro de la normalidad (19 segundos): en ausencia de heparina en la muestra o en el tratamiento con una correcta extracción de la muestra. Tras ello se realizan pruebas específicas para descartar la presencia de un anticoagulante circulante: en primer lugar se realizó un screening de anticoagulante lúpico (AL) utilizando los reactivos LA1/LA2 de Dade-Behring y LACS/LACC de Izasa, que aportaban una fuente de fosfolípidos externa, realizándose una determinación basal, y otra tras la mezcla con pool de plasma normal, siendo los resultados negativos para la presencia de anticoagulante lúpico. Debido a ello, fue preciso comprobar la existencia de otro tipo de anticoagulante circulante o inhibidores. Asímismo, se llevaron a cabo estudios de mezcla para el APTT, inmediata e incubadas 2 horas a $37^{\circ} \mathrm{C}$, con el fin de descartar la presencia de algún inhibidor de los factores de la vía intrínseca. Se comprobó la normalización de los tiempos en segundos y ratio, tras las mezclas de plasma, descartándose, por tanto, la presencia de inhibidor de factores de dicha vía.

Se llevó a cabo el mismo procedimiento de mezclas de plasma para el estudio del alargamiento del tiempo de trombina, evidenciándose una falta de corrección del mismo tras la incubación. Este hallazgo confirma la presencia de un inhibidor de la trombina, con un comportamiento similar a la heparina (alargamiento del TT con un TR normal).

Orina de 24 h: proteinuria inicial 18,4 g/día, con proteínas totales de $8,4 \mathrm{~g} / \mathrm{dl}$, albuminemia de $1,85 \mathrm{~g} / \mathrm{dl}$ con aumento de las alfa-2-globulinas e hipergammaglobulinemia heteroclonal. Al décimo día de tratamiento la proteinuria era de $3 \mathrm{~g}$ /día y de $320 \mathrm{mg} 3$ meses des-

TABLA I

\section{EVOLUCIÓN DE PARÁMETROS ANALÍTICOS MÁS} SIGNIFICATIVOS

\begin{tabular}{lcccccc}
\hline Fecha & TGP & F. Alcalinas & Colesterol & Proteinuria & RPR & APTT \\
\hline Inicial & 116 & 1344 & 377 & 18.4 & $1 / 32$ & 64 \\
Control & 17 & 118 & 160 & 0.32 & 0 & 44 \\
\hline
\end{tabular}

pués. En muestra de orina se objetiva microhematuria. La radiografía de tórax, el electrocardiograma y ecocardiograma fueron normales. En la eco abdominal se aprecia hepatomegalia, sobre todo a expensas del lóbulo derecho, de ecogenicidad heterogénea, con múltiples lesiones focales hipoecoicas, de aproximadamente $1 \mathrm{~cm}$ de diámetro (Fig. 1), junto a otra mayor de $1.64 \mathrm{~cm}$, lesión compatible con hemangioma por RNM. Se practicó PAAF, guiada por eco, de un nódulo hepático. La RNM demuestra la desaparición de los nódulos al décimo día de tratamiento, lo que se confirma con la eco a las 6 semanas de completar el mismo (Fig. 2). Nefrolitiasis bilateral multicéntrica.

Tras la realización de la PAAF hepática, que reveló reacción inflamatoria inespecífica y ausencia de espiroquetas, presentó ligera anemización, con descenso del hematocrito en 6 puntos. Tras ello no se consideró realizar una punción lumbar.

Los estudio inmunológicos fueron negativos salvo ANA 1/160 y anticuerpos antimitocondriales $1 / 80$. Los niveles de crioglobulinas, C3 y C4 fueron normales.

El tratamiento indicado fue penicilina $\mathrm{G}$ sódica a dosis de 4 millones de unidades cada 6 horas durante 2 semanas, iniciando

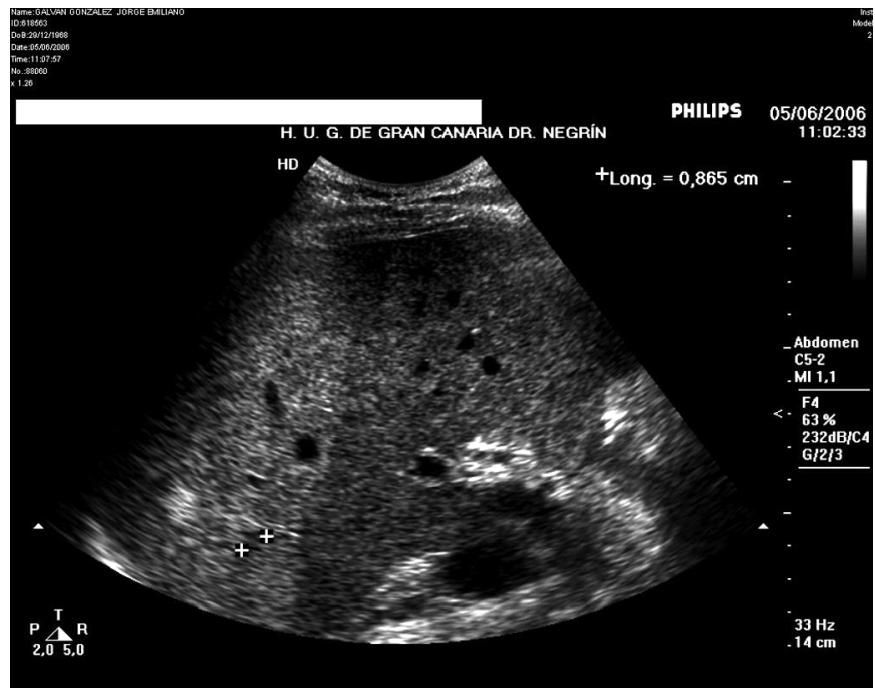

Fig. 1. Ecografía hepática (antes del tratamiento): Se aprecian múltiples lesiones focales.

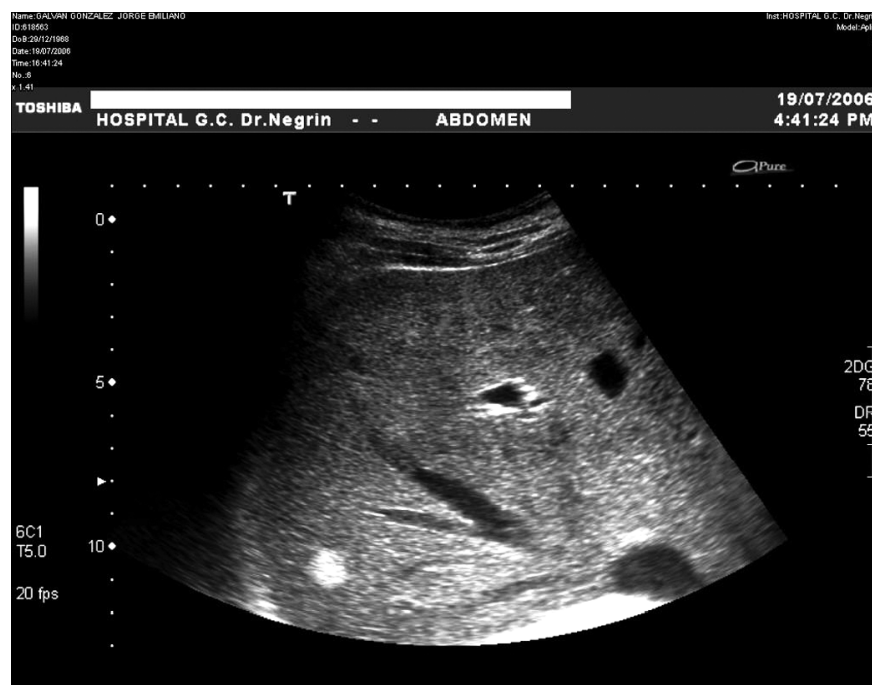

Fig. 2. Ecografía hepática: Seis semanas después del tratamiento se aprecia la desaparición de las lesiones focales hipoecoicas, con persistencia del hemangioma. 
dosis crecientes, como profilaxis de la reacción de Jarisch-Herxheimer. Fue dado de alta asintomático, circunstancia que persistía en una revisión 3 meses después, momento en que la RPR se había negativizado y seguía negativa la serología para los VIH 1 y 2 . El estudio de coagulación realizado a los 3 meses sólo mantenía mínima elevación del APTT. Se le permitió posteriormente completar cuidados odontológicos, no presentando sangrado anormal.

Discusión: El caso presentado reúne múltiples criterios clínicos de escasa frecuencia en la lúes secundaria, como son el síndrome nefrótico, la hepatitis colestática y el cuadro meníngeo (9-14), frente a las clásicas manifestaciones muco-cutáneas $(1,2)$, con criterios serológicos bien definidos, y una respuesta clara a la penicilina. Si a ello le añadimos la presencia de LOES hepática y un anticoagulante circulante (3-8), que desaparece tras el tratamiento, todo ello configura una exepcional presentación, única hasta el momento en toda la literatura analizada. Ninguno de los 4 casos encontrados en la literatura con anticoagulante circulante (3-6) presentó todas las manifestaciones de este caso, y ninguno de ellos mostró manifestaciones clínicas transitorias de sangrado.

En relación con LOES hepáticas y sífilis secundaria sólo hemos encontrado 2 publicaciones, una de ellas muy reciente y con una iconografía menos ilustrativa, al margen que dicho caso coexistía con serología retrovírica positiva $(7,8)$. El carácter inflamatorio e inespecífico de la PAAF de una LOE hepática, con las pruebas complementarias existentes en el momento de su realización afirman que la misma podía haberse evitado, máxime cuando no pudo demostrarse la presencia de espiroquetas. De algún modo se realiza una prueba que determinó un cierto riesgo al paciente, con caída del hematocrito, de escasa utilidad, circunstancia que también habían practicado previamente en el caso antes comentado (8). La aportación de la anatomía patológica en la lúes secundaria es inespecífica, ya que es muy raro poder demostrar espiroquetas a nivel hepático o cutáneo (15-17). Probablemente a nivel hepático el estudio se hace buscando otro diagnóstico, que fue lo que realmente ocurrió en este caso.

La evolución del síndrome nefrótico en 3 meses hacia la casi desaparición de la proteinuria y la normalización de lípidos y albúminemia, establece una clara relación con nefropatía asociada a la lúes secundaria, que desaparece al curar la infección $(10,11)$. No se consideró la realización de biopsia renal para demostrar dicho proceso. La coexistencia con microhematuria, interpretada inicialmente en el contexto de glomerulopatía, o por la urolitiasis, y su desaparición posterior, sugieren poder tener relación con el anticoagulante circulante, al igual que la ligera anemizacion tras la biopsia hepática o el sangrado intenso que presentó tras una limpieza dental rutinaria en el curso del cuadro clínico. No se puede descartar que tanto la microhematuria como la discreta elvación inicial de la creatinina pudiesen tener relación con la glomerulopatía. Fuese lo que fuese la normalización posterior establece un claro nexo casual con la sífilis.
Respecto a la reacción meníngea en el secundarismo luético y en la lúes terciaria se han hecho múltiples comunicaciones al respecto (2). El paciente presentó clínica compatible con reacción meningea. No se consideró la punción lumbar, ante la diátesis hemorrágica, mostrada por la PAAF hepática, y la mejoría con el tratamiento determinó el no realizarla.

El marcado cambio observado en 3 meses en los lípidos, llámese colesterol total inicial de $377 \mathrm{mg} / \mathrm{dl}$ y control posterior de 160 , se explica por tener una etiología dual secundaria: colestasis total y marcado síndrome nefrótico (18). La resolución de estos problemas conlleva la desaparición de la hiperlipemia. Otros cambios bioquímicos transitorios que se observaron en la analítica inicial como son discreta elevación de la creatinina y bilirrubina y la presencia de hipergammaglobulinemia heteroclonal se explican en el contexto global de la enfermedad infecciosa con perfil sistémico, y, desaparecieron al curar el proceso.

Podría cuestionarse el tratamiento, al seguir una pauta de lúes terciaria en el secundarismo luético. La respuesta clínica, serológica y bioquímica espectacular deberían replantear esta alternativa si el cuadro sistémico tuviese un perfil tan marcado. En cuanto a incrementar progresivamente la dosis de penicilina para evitar la reación de Jarisch-Herxheimer existen comentarios históricos en relación con la fiebre tifoidea y el cloramfenicol. Nosotros ya lo habíamos iniciado previamente cuando un paciente nos presentó la reacción de modo cataclísmico (19), al iniciar dosis elevadas, como se preconiza en los libros de texto, y, desde entonces lo hacemos de modo ascendente cuando iniciamos el tratamiento de una lúes terciaria o cuaternaria, sin haber observado de nuevo dicha reacción.

En resumen presentamos un caso de lúes secundaria con múltiples manifestaciones clínicas poco frecuentes, donde lo más expresivo y poco conocido previamente son las LOES hepáticas y junto a ello la rareza del anticoagulante circulante con expresión clínica de diátesis hemorrágica. La respuesta global al tratamiento con penicilina sugiere que en presencia de un proceso explicado por infecciones de esta índole con diagnóstico microbiológico es preferible indicar tratamiento y ver respuesta que realizar pruebas complementarias de riesgo, como en el caso comentado, la PAAF hepática, ya que el diagnóstico serológico, al menos cuando los niveles reagínicos para lúes son elevados, es concluyente para iniciar tratamiento y ver respuesta antes de realizar otras pruebas.

Del caso comentado podemos sacar algunas conclusiones, como ser partícipe del reciente incremento comunicado de incidencia de lúes (20-22), la presentación clínica sistémica de la lúes secundaria, la espectacular respuesta a la penicilina en todos los estadíos y el carácter proteiforme de esta enfermedad, que en el estadío secundario puede no presentar de un modo definido hasta en un $25 \%$ las manifestaciones muco-cutáneas $(2,23)$.

\section{Bibliografía}

1. Martín Ruiz C, Pascua Molina J. Infecciones por espiroquetas. Medicine. Unidad temática 2006; 55: 3563-71.

2. Lukehart SA. Sífilis. En: Brawnwald E, Stephen H, Fauci AS, Longo DL, Kasper DL, Jameson JL, editors. Harrison. Principios de Medicina Interna (ed. Española). Madrid: Mc Graw Hill; 2003. p. 1233-42.

3. Congy F, Moulias R, Beaufils H, Rujeau JC, Sqalli S, Debat P, Loeper J. Syndrome Néphrotique, hépatite et anticoagulant circulant au cours d'une syphilis secondaire. Ann Med Interne (Paris) 1977; 128: 799-802.

4. Guillaume JC, Gouault-Heilmann M, Dumont MD, Touraine R. [Occurrence of lupus-type circulating anticoagulant in syphilis (author's transl)]. Ann Dermatol Venereol 1981; 108: 139-43.

5. Feldmann JL, Menkes CJ, Lacombe MJ, Delbarre F. [Humeral lytic lesions and circulating anticoagulant in secondary syphilis (author's trans1)]. Nouv Presse Med. 1979; 8: 4044-5.

6. Arlet P, Botreau Y, Duffaut M, Le Tallec Y. Circulating anticoagulant during primosecondary syphilis and Q fever. Nouv Presse Med 1981; 10: 2909

7. Mahto M, Mohammed F, Wilkins E, Mason J, Haboubi NH. Pseudohepatic tumor associated with secondary syphilis in an HIV-positive male. Int J STD AIDS 2006; 17: 139-41.

8. Vas W, Thomasson J, Tang-Barton P, Salimi Z, Catral B. Computed tomography and ultrasound appearance of liver disease in secondary syphilis. Diagn Imaging Clin Med 1985; 54: 1-6.

9. Mullick CJ, Liappis AP, Benator DA, Roberts AD, Parenti DM. Syphilitic hepatitis in HIV-infected patients: A repot of seven cases and review of the literature. Clin Infect Dis 2004; 39: 100-5. 
10. Ramos RM, Ramos JM, Barrio M, Torralba M, Herrero F. Síndrome nefrótico asociado a sífilis secundaria. Enferm Infecc Microbiol Clin 2000; 18: 421-2.

11. Koenig M, Duband S, Thibaudin D, Cathebras P. Rash and nephrotic syndrome: consider syphilis. Presse Med 2005; 34: 657.

12. Ridruejo E, Mordoh A, Herrera F, Avagnina A, Mando OO. Severe cholestatic hepatitis as the first symptom of secondary syphilis. Dig Dis Sci 2004; 49: 1401-4.

13. Gómez Martínez MV, Gallardo FG, Cobo Soler J, Babe Romero J, Ríos Ferreiros M, Valencia Ortega E. Osteítis en la sífilis secundaria. Rev Esp Med Nucl 2003; 22: 424-6.

14. Mannara GM, Sacilotto C, Frattasio A, Pedace E, Di Loreto C, Ferlito A. Bilateral secondary syphilis of the tonsil. J Laryngol Otol 1999; 113 : $1125-7$.

15. LOE HEPÁTICA. Brooks SE, Hanchard B, Terry S, Audretsch JJ. Hepatic ultraestructure in secondary syphilis. Arch Pathol Lab Med 1979; 103: 451-5.

16. Hoang MP, High WA, Molberg KH. Secondary syphilis: a histologic and immunohistochemical evaluation. J Cutan Pathol 2004; 31: 595-9.
17. Wenhai L, Jianzhong Z, Cao Y. Detection of Treponema pallidum in skin lesions of secondary syphilis and characterization of the inflammatory infiltrate. Dermatology 2004; 208: 94-7.

18. Hiperlipoproteinemias. En: MKSAP 13. Endocrinología y Metabolismo. Ed: ACP y SEMI (versión española) Madrid: Drug Farma SL; 2006. p. 21-6.

19. Suárez Ortega S, Lafarga B, Melado Sánchez P, Artiles Vizcaíno J, Conde Martel A, Betancor León P. Accidente cerebrovascular isquémico, neurosífilis e infección por el VIH. Canarias Médica 1999; 14: 67-9.

20. Marcus U, Hamouda O. Syphilis in Germany: 2004: Diagnoses increasing, particularly in smaller cities and rural areas. Euro Surveill 2005: 10 (7): E050728.3.

21. Payne L, Berglund T, Henriksson L, Berggren-Palme I. Re-emergence of syphilis in Sweden: Results from a surveillance study for 2004. Euro Surveill 2005; 10: E051110.2.

22. Young F. Syphilis: Still with us, so watch out! J Fam Health Care 2006; 16: 77-81.

23. Thein M, Acland K, Lightman S, Lynn W. Secondary syphilis: A multisystem disease not to be forgotten. Hosp Med 2005; 66: 178-9. 